\title{
Prefrontal-Temporal Disconnection Impairs Recognition Memory but Not Familiarity Discrimination
}

\author{
Philip G.F. Browning, ${ }^{1,2}$ Mark G. Baxter, ${ }^{1}$ and David Gaffan ${ }^{2}$ \\ ${ }^{1}$ Glickenhaus Laboratory of Neuropsychology and Friedman Brain Institute, Department of Neuroscience, Mount Sinai School of Medicine, New York, \\ New York 10029, and 2Department of Experimental Psychology, South Parks Road, Oxford University, Oxford, OX1 3UD, United Kingdom
}

\begin{abstract}
Neural mechanisms in the temporal lobe are essential for recognition memory. Evidence from human functional imaging and neuropsychology, and monkey neurophysiology and neuropsychology also suggests a role for prefrontal cortex in recognition memory. To examine the interaction of these cortical regions in support of recognition memory we tested rhesus monkeys with prefrontalinferotemporal (PFC-IT) cortical disconnection on two recognition memory tasks, a "constant negative" task, and delayed nonmatchingto-sample (DNMS). In the constant negative task monkeys were presented with sets of 100 discrimination problems. In each problem one unrewarded object was presented once every day, and became familiar over the course of several days testing. The other, rewarded object was always novel. In this task monkeys learned to avoid the familiar constant negatives and choose the novel objects, so performance on this task is guided by a sense of familiarity for the constant negatives. Following PFC-IT disconnection monkeys were severely impaired at reacquiring the rule (to avoid familiar items) but were subsequently unimpaired at acquiring new constant negative problems, thus displaying intact familiarity recognition. The same monkeys were impaired in the acquisition of the DNMS task, as well as memory for lists of objects. This dissociation between two tests of recognition memory is best explained in terms of our general hypothesis that PFC-IT interactions support the representation of temporally complex events, which is necessary in DNMS but not in constant negative. These findings, furthermore, indicate that stimulus familiarity can be represented in temporal cortex without input from prefrontal cortex.
\end{abstract}

\section{Introduction}

Interactions between prefrontal cortex (PFC) and inferior temporal cortex (IT) are essential for a variety of complex memory functions (Fuster et al., 1985; Gaffan et al., 2002; Browning et al., 2005). In monkeys, one-way to test which aspects of memory require this interaction is to surgically disconnect PFC from IT, by combining a unilateral lesion of PFC in one hemisphere with a unilateral lesion of IT in the other hemisphere (PFC-IT disconnection). IT is essential for the identification of objects (Tanaka, 1996) and so this surgical disconnection impairs cognitive tasks that depend upon PFC having access to visual information represented in IT. PFC-IT disconnection impairs visual conditional learning, object discrimination learning set and other complex strategy tasks (Parker and Gaffan, 1998a, b; Bussey et al., 2002; Browning et al., 2007; Wilson and Gaffan, 2008). We have hypothesized that the underlying impairment common to these tasks is a deficit in the representation of temporally complex visual events (Browning and Gaffan, 2008a, b; Wilson et al., 2010). We previously isolated the requirement to represent tem-

Received Dec. 17, 2012; revised April 22, 2013; accepted April 27, 2013.

Author contributions: P.G.F.B. and D.G. designed research; P.G.F.B. and M.G.B. performed research; P.G.F.B. analyzed data; P.G.F.B. wrote the paper.

This research was supported by the Medical Research Council (D.G. and P.G.F.B.) and the Wellcome Trust (M.G.B.) We thank P.L.Croxson, C.R.E. Wilson, K.L. Murphy, and G. Daubney for technical assistance. We thankE.A. Murray for comments on an earlier version of the paper.

Correspondence should be addressed to Dr Philip G.F. Browning, Department of Neuroscience, Mount Sinai School of Medicine, One Gustave L Levy Place, Box 1065, New York, NY 10029. E-mail: philip.browning@mssm.edu. DOI:10.1523/JNEUROSCI.5759-12.2013

Copyright $\odot 2013$ the authors $\quad 0270-6474 / 13 / 339667-08 \$ 15.00 / 0$ porally complex events using several variations of a sequence discrimination task. Monkeys with PFC-IT disconnection are impaired in object-reward association learning only when the stimuli are extended in time to form multi-item serial visual compounds. In contrast, simple, single-item object discrimination tasks are unimpaired even when the reward is delayed (Parker and Gaffan, 1998b; Gaffan et al., 2002; Browning and Gaffan, 2008a). This shows that learning about sequences of visual events engages PFC-IT interactions in a qualitatively different way than learning that does not require linking of temporally separate visual events.

This analysis of prefrontal function is supported by studies that compared temporally complex and nontemporally complex versions of otherwise cognitively similar tasks.

For example, object-reward association learning is unimpaired following prefrontal-temporal disconnection if problem pairs are presented concurrently, but learning is impaired if the problems are presented serially as single pairs allowing for the development of a discrimination learning set (Browning et al., 2007). Discrimination learning set relies on the linking together of choices and outcomes with the same problem in close temporal proximity, something which does not happen with concurrently presented problem pairs (Murray and Gaffan, 2006). Similarly, object reversal learning is unimpaired following PFC-IT disconnection if reversal problems are presented concurrently but is severely impaired if problems are presented serially (Wilson and Gaffan, 2008). These dissociations, combined with the evidence from visual sequence learning, suggest that the temporal complexity of a task determines whether PFC-IT interac- 
tions are required. The present experiment examined whether the same analysis could be applied to recognition memory-the ability to judge prior occurrence. Both PFC-IT disconnection and bilateral PFC lesions impair recognition memory as evidenced by impairment on delayed matching-to-sample (DMS) (Bachevalier and Mishkin, 1986; Parker and Gaffan, 1998a). It is important to examine therefore whether the impairments in recognition memory tasks following prefrontal lesions represent true deficits in recognition memory or whether they arise because those tasks require the representation of temporally complex events.

We designed a novel test of recognition memory that can be performed independently of the requirement to represent temporally complex events. In Experiment 1, monkeys were presented with sets of 100 discrimination problems in which they chose between two objects. In each problem one unrewarded object was presented once every day, which became familiar over the course of several days testing. The other, rewarded object was always a novel object that the monkey had never seen previously. Monkeys learned to avoid the familiar "constant negatives" and choose instead the novel objects presented. Performance on the constant negative task was guided by a sense of familiarity for the constant negatives. After preoperative training, monkeys either received PFC-IT disconnection or rested for an equivalent period of time (controls) and were presented with retention tests as well as new sets of constant negatives to learn. In Experiment 2, the same monkeys were trained postoperatively to acquire delayed nonmatching-to-sample (DNMS). Impairment in both tasks would suggest a general role for prefrontal cortex in performing judgments of prior occurrence. However, it was our prediction that monkeys with PFC-IT disconnection would not be impaired on the constant negative task because that task does not require the representation of temporally complex events.

\section{Materials and Methods}

\section{Subjects}

All experiments were conducted in accordance with UK Home Office regulations. Seven experimentally naive male rhesus monkeys (Macaca mulatta) of mean age 5 years 2 months and of mean weight $6.1 \mathrm{~kg}$ at the start of training were used. Monkeys were all housed together in large enclosures with water provided ad libitum. Monkeys were pretrained to respond to the touchscreen and to retrieve rewards before starting the experimental tasks. In Experiment 1 monkeys were preoperatively trained on the constant negative task before being allocated to an operated group or an unoperated control group, balanced for learning proficiency. Four monkeys (CON1-4) formed the control group and three monkeys (OP1-3) formed the operated group. Each monkey was operated on twice to achieve PFC-IT disconnection; once to remove the IT unilaterally and again to remove PFC unilaterally in the opposite hemisphere. The order of lesions and the choice of hemisphere for each lesion were counterbalanced as best possible between the three operated monkeys. Operated monkeys rested for 2 weeks following each surgery to allow for good recovery. Control monkeys were rested for equivalent periods of time at the appropriate point in testing to control for the break in testing experienced by operated monkeys. In Experiment 1 monkeys were tested between surgeries to gather control data for the effect of unilateral lesions, and tested again after the second surgery to assess the effects of PFC-IT disconnection. Monkeys began Experiment 2 immediately after Experiment 1.

\section{Apparatus}

The apparatus consisted of a computer-controlled touch-sensitive monitor ( $380 \mathrm{~mm}$ wide by $280 \mathrm{~mm}$ high) on which the stimulus material was presented. The monkey sat in a wheeled transport cage $150 \mathrm{~mm}$ from the screen and made choices between stimuli by reaching out through the bars of the cage. Touches were registered by the computer and banana flavored pellets (190 mg, Noyes) were delivered as rewards for correct responses to a food hopper placed centrally below the monitor. A single large food reward was delivered at the end of each session by the opening of a box set to one side of the centrally placed food hopper. The opening of the box with the large food reward, like all other aspects of the events and the experimental contingencies during any session of training, was under computer control.

\section{Stimulus material}

The stimuli used were colored clipart “objects", $128 \times 128$ pixels in size presented on a white background on the touchscreen that had a resolution of 800 pixels wide by 600 pixels high. Objects were $\sim 60 \times 60 \mathrm{~mm}$ square and were presented centrally or on the left or right side of the screen $50 \mathrm{~mm}$ from the edge. The monkey chose between the objects by touching one of them. Each constant negative was chosen without replacement from a pool of 1000 unique clipart objects. Novel objects were chosen without replacement from a separate pool of 19,100 unique clipart objects. Each monkey was presented with objects selected according to a different random seed so that each monkey saw a different selection of objects throughout the task.

\section{Experiment 1: constant negative}

Task procedure. In a daily session, monkeys worked through a set of 100 unique discrimination problems in which they chose between two objects, one presented on the left side of the screen and one on the right. In each problem one object was designated the unrewarded constant negative stimulus $(\mathrm{S}-)$ and one the rewarded stimulus $(\mathrm{S}+)$. The $\mathrm{S}-$ was presented once every day and became familiar over the course of several days testing. The $\mathrm{S}+$ presented alongside the $\mathrm{S}-$ was always a novel object that the monkey had never seen previously and once used it was discarded from the image pool. Monkeys learned to avoid the familiar constant negatives and choose instead the novel objects presented. The order of presentation of constant negatives within a session and the left/ right positions of the $\mathrm{S}+$ and $\mathrm{S}-$ were randomized. Touching the $\mathrm{S}+$ resulted in the delivery of a single reward pellet; both objects remained on the screen for a further $500 \mathrm{~ms}$ and then disappeared. Touching the Sresulted in no reward and both objects disappeared immediately. The intertrial interval began as soon as the choice stimuli disappeared from the screen. The intertrial interval (ITI) was $10 \mathrm{~s}$ following a correct response or $20 \mathrm{~s}$ following an incorrect response. Any touches to the screen during the ITI reset the interval. On the last trial of the session a correct response opened the box containing the large food reward (see Apparatus). An incorrect response resulted in the $20 \mathrm{~s}$ ITI followed by a forced choice trial in which a single novel object was presented. A response to this object opened the box thus ensuring that monkeys always obtained the large food reward. Monkeys learned each set of 100 problems to a criterion of two consecutive sessions of $90 \%$ correct or greater. Once criterion was reached monkeys began the next set the following testing day.

Training procedure. Monkeys learned several sets of 100 problems over the course of the experiment. To acquire the task principle and adjust to the demands of the task, monkeys initially learned a single set of 100 constant negative problems. For this training set only, monkeys were presented with problems $1-25$ for 10 sessions, followed by problems $1-50$ for 10 sessions and finally problems $1-100$, which were learned to criterion. Data from this training set was not used in the experiment. All subsequent sets (sets 1-7) were presented as 100 problem sets so that the exposure to each problem within a set was identical. The basic experimental design was as follows. Monkeys learned three consecutive preoperative sets (sets 1-3) at which point monkeys were assigned to the operated group or the control group (see Subjects). Monkey learned set 4 following the first surgery (interoperative) and sets 5-7 following prefrontal-temporal disconnection (postoperative). The number of errors to criterion constituted the dependent measure in acquisition of constant negative problems. To assess retrieval monkeys performed retention tests with sets $1-3$ before the first surgery, after unilateral surgery, after the final surgery to complete the disconnection, and finally at the end of the experiment following acquisition of set 7 . Retention tests consisted of the presentation of a single set of problems in an identical manner as in the acquisition phase. Thus, after acquiring sets 1-3 preop- 
Table 1. Performance of control (CON) and operated (PFCxIT) monkeys in constant negative

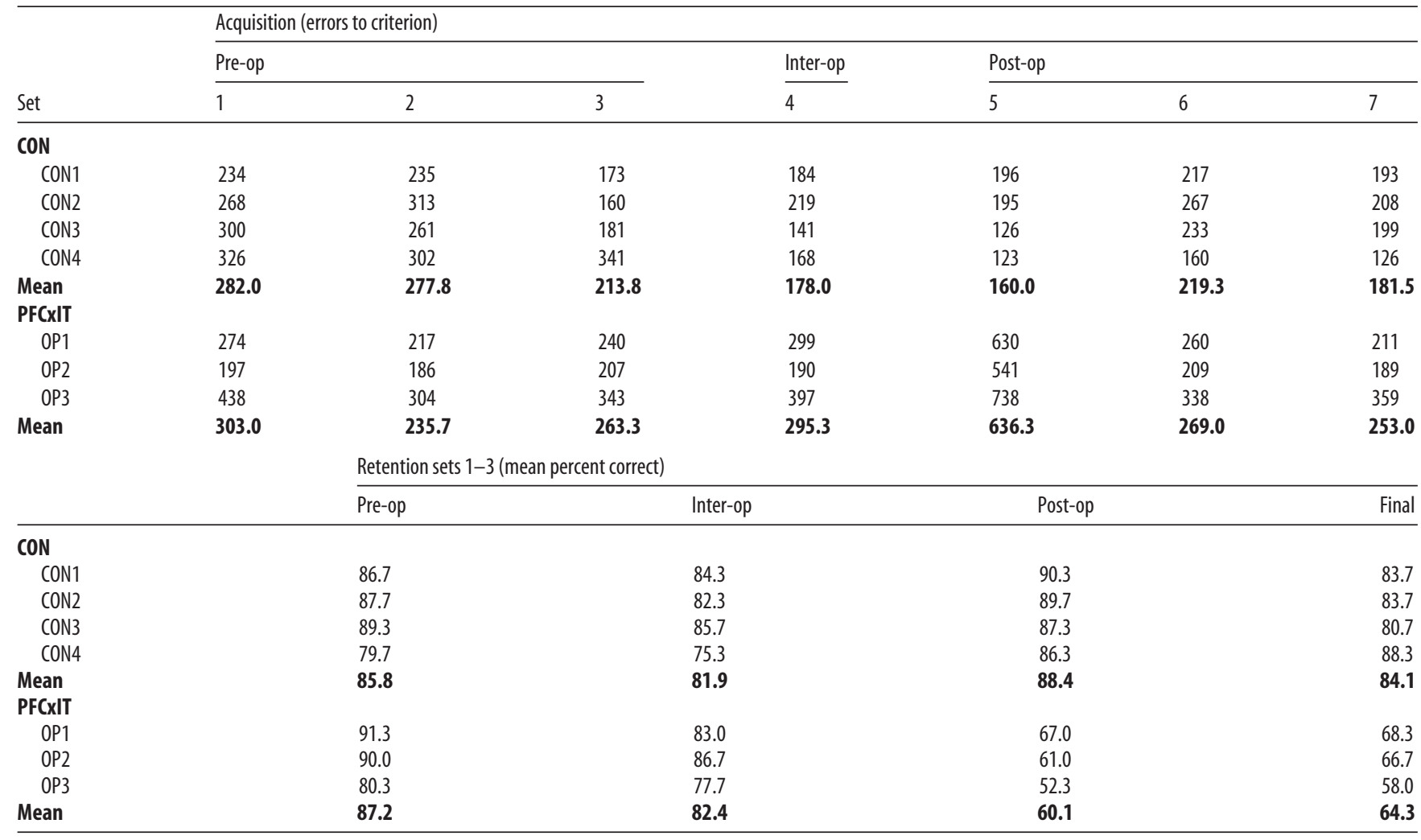

Top panel, Acquisition data (errors to criterion) from the three phases of the experiment, pre-op, inter-op and post-op. Bottom panel, Retention (mean percent correct) of all preoperatively learned problems was measured immediately before surgery (Pre-op), between unilateral surgeries (Inter-op), immediately after final surgery to complete the disconnection (Post-op), and finally after completion of the post-op acquisition phase.

eratively, monkeys completed one session of set 1 , followed by one session of set 2 and then one session of set 3 and the percentage error score made in these sessions constituted the preoperative retention test score (Table 1). Monkeys repeated this retention test immediately after the first and second surgeries and at the end of the experiment.

Experiment 2: DNMS

Task procedure. Experiment 2 compared the postoperative acquisition and performance of DNMS with trial unique objects. The basic task procedure was identical to that of Browning et al. (2010) except that the objects were chosen from the pool of 20,100 images excluding those already used in Experiment 1 (see Stimulus material). Each trial consisted of the presentation of the sample object in the center of the touchscreen and the monkey touched it to proceed. Touching the sample caused it to disappear $500 \mathrm{~ms}$ later. After a $2 \mathrm{~s}$ delay two choice stimuli appeared on the left and right positions on the screen (vertically centered, $50 \mathrm{~mm}$ from the edge of the screen) one which was the sample object and one which was a novel object, and the monkey was rewarded with a food pellet for touching the novel choice stimulus. After a correct response both objects remained on the screen for $500 \mathrm{~ms}$. After an incorrect response, both objects disappeared immediately. The intertrial interval began as soon as the choice stimuli disappeared from the screen. At this initial stage of training the ITI was $8 \mathrm{~s}$ following a rewarded response and $16 \mathrm{~s}$ following an unrewarded response and this interval was reset by touches to the screen during this time. For the final trial in the session if the monkeys chose incorrectly, an additional trial was presented until the monkey chose correctly, thus ensuring that the large food reward would always be obtained.

Monkeys were then trained with lists of samples in the following manner. Several samples were presented one after another while monkeys touched each object in the list to proceed to the next. The delay between sample objects was $5 \mathrm{~s}$. Having worked through the list of samples, monkeys worked through the list of choices. The presentation of lists of choice stimuli was in reverse order to the list of samples such that the hypothetical alphabetical list of samples A though $\mathrm{E}$ would be followed by choices
E versus N1, D versus N2, C versus N3, B versus N4, A versus N5 where N1-N5 are always novel objects. As throughout the experiment the novel choice object was rewarded. The ITI after a choice trial in sessions with lists was $10 \mathrm{~s}$ regardless of the outcome of the trial and this was not reset by touches to the screen during this time.

Training procedure. Monkeys were trained on the basic task with $2 \mathrm{~s}$ delays to a criterion of $90 \%$ correct choices in two consecutive sessions of 100 trials. Following this, monkeys were given performance tests with longer lists of samples and choices. Monkeys performed sessions of list lengths of 2 increasing to list lengths of 3 and then 5 if they performed at $90 \%$ correct in a day. At each list length test, failure was determined if subjects completed 500 trials without reaching criterion. Thus each subject was tested on higher list lengths either after reaching criterion or after reaching 500 trials in any list length test. This procedure was used so that regardless of the operated monkey's initial failure at LL2, performance information could be obtained about the rate of forgetting during longer lists.

\section{Surgical procedures}

Neurosurgical procedures were performed in a dedicated operating theater under aseptic conditions. Steroids (methylprednisolone, $20 \mathrm{mg} / \mathrm{kg}$, i.m.) were given the night before surgery, and 3 doses were given $4-6 \mathrm{~h}$ apart (i.v. or i.m.) on the day of surgery to protect against intraoperative edema and postoperative inflammation. The monkey was sedated on the morning of surgery with both ketamine $(10 \mathrm{mg} / \mathrm{kg})$ and xylazine $(0.25-$ $0.5 \mathrm{mg} / \mathrm{kg}$, i.m.). Once sedated, the monkey was given atropine $(0.05$ $\mathrm{mg} / \mathrm{kg}$ ) to reduce secretions, antibiotic (amoxicillin, $8.75 \mathrm{mg} / \mathrm{kg}$ ) for prophylaxis of infection, opioid (buprenorphine $0.01 \mathrm{mg} / \mathrm{kg}$, i.v., repeated twice at $4-6 \mathrm{~h}$ intervals on the day of surgery, i.v. or i.m.) and nonsteroidal anti-inflammatory (meloxicam, $0.2 \mathrm{mg} / \mathrm{kg}$, i.v.) agents for analgesia, and an $\mathrm{H} 2$ receptor antagonist (ranitidine, $1 \mathrm{mg} / \mathrm{kg}$, i.v.) to protect against gastric ulceration as a side effect of the combination of steroid and nonsteroidal anti-inflammatory treatment. The head was shaved and an intravenous cannula put in place for intraoperative delivery of fluids (warmed sterile saline drip, $5 \mathrm{ml} / \mathrm{h} / \mathrm{kg}$ ). The monkey was 
moved into the operating theater, intubated, placed on sevoflurane anesthesia (1-4\%, to effect, in $100 \%$ oxygen), and then mechanically ventilated. A hot air blower (Bair Hugger) allowed maintenance of normal body temperature during surgery. Heart rate, oxygen saturation of hemoglobin, mean arterial blood pressure, and tidal $\mathrm{CO}_{2}$, body temperature, and respiration rate were monitored continuously throughout surgery. In each surgery, the monkey was placed in a head holder and the head cleaned with alternating antimicrobial scrub and alcohol and draped to allow a midline or coronal incision. The skin and underlying galea were opened in layers. When the lesion was complete, the dura was sewn over the lesion site, the bone flap replaced and held with loose sutures, and the skin and galea were closed in layers. The monkey was removed from the headholder and anesthesia discontinued. The monkey was extubated when a swallowing reflex was observed, returned to the home cage, and monitored continuously until normal posture was regained (usually within 10 $\mathrm{min}$ ). Nonsteroidal anti-inflammatory analgesic (meloxicam, $0.2 \mathrm{mg} / \mathrm{kg}$, oral) and antibiotic (amoxicillin, $8.75 \mathrm{mg} / \mathrm{kg}$, oral) treatment continued following surgery in consultation with veterinary staff, typically for $5 \mathrm{~d}$. Operated monkeys that were socially housed rejoined their social groups as soon as practicable after surgery, usually within $3 \mathrm{~d}$ of the operation.

\section{Unilateral prefrontal ablation}

In the hemispheres with prefrontal ablation, the intention was to remove prefrontal cortex while sparing cingulate, supplementary, and premotor cortex. The posterior limit of the lesion on the lateral surface of the frontal lobe followed an approximate line joining the tips of the ascending and descending limbs of the arcuate sulcus and the posterior end of the principal sulcus. The entire arcuate sulcus was to be spared. From the anterior-most point of the ascending limb of the arcuate sulcus the boundary extended medially and then ventrally into the interhemispheric fissure. Medially this boundary remained at this same anterior-posterior level up to the crown of the cingulate sulcus. The entire cingulate cortex was to be spared, including both banks of the cingulate sulcus. All cortex forward of this boundary was removed, including the cortex in both banks of the principal sulcus. From the anterior-most point of the descending limb of the arcuate sulcus the boundary extended downward to the lateral sulcus. The entire orbital surface of the frontal lobes was removed including the cortex in the medial and lateral orbital sulci. This orbital removal was extended onto the ventral part of the medial surface of the hemisphere as far dorsally as the rostral sulcus. The white matter surrounding the corpus striatum and the striatum itself was to be left intact.

\section{Unilateral inferior temporal cortex ablation}

The boundaries of the unilateral inferotemporal ablation were identical to those in previous experiments with this lesion in our laboratory. The arch of the zygoma was removed and the temporal muscle was detached

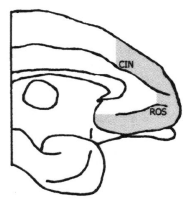

UNILATERAL PREFRONTAL ABLATION
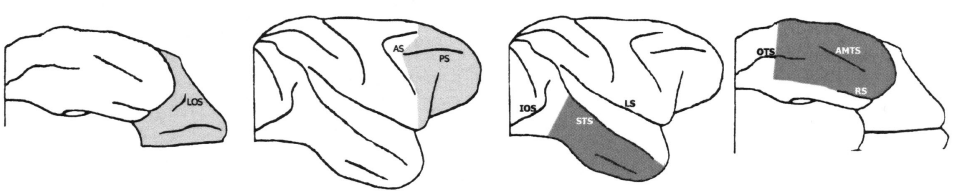

Figure 1. Intended extents of the unilateral cortical ablations in Group PFCXIT shown from medial, ventral, and lateral views. The shaded areas indicate the areas of intended removal. CIN, Cingulate sulcus; ROS, rostral suclus; LOS, lateral orbital sulcus; PS, principal suclus; AS, arcuate sulcus; OTS, occipitotemporal sulcus; AMTS, anterior middle temporal sulcus; RS, rhinal suclus; IOS, intraoccipital sulcus; STS, superior temporal suclus; LS, lateral sulcus.

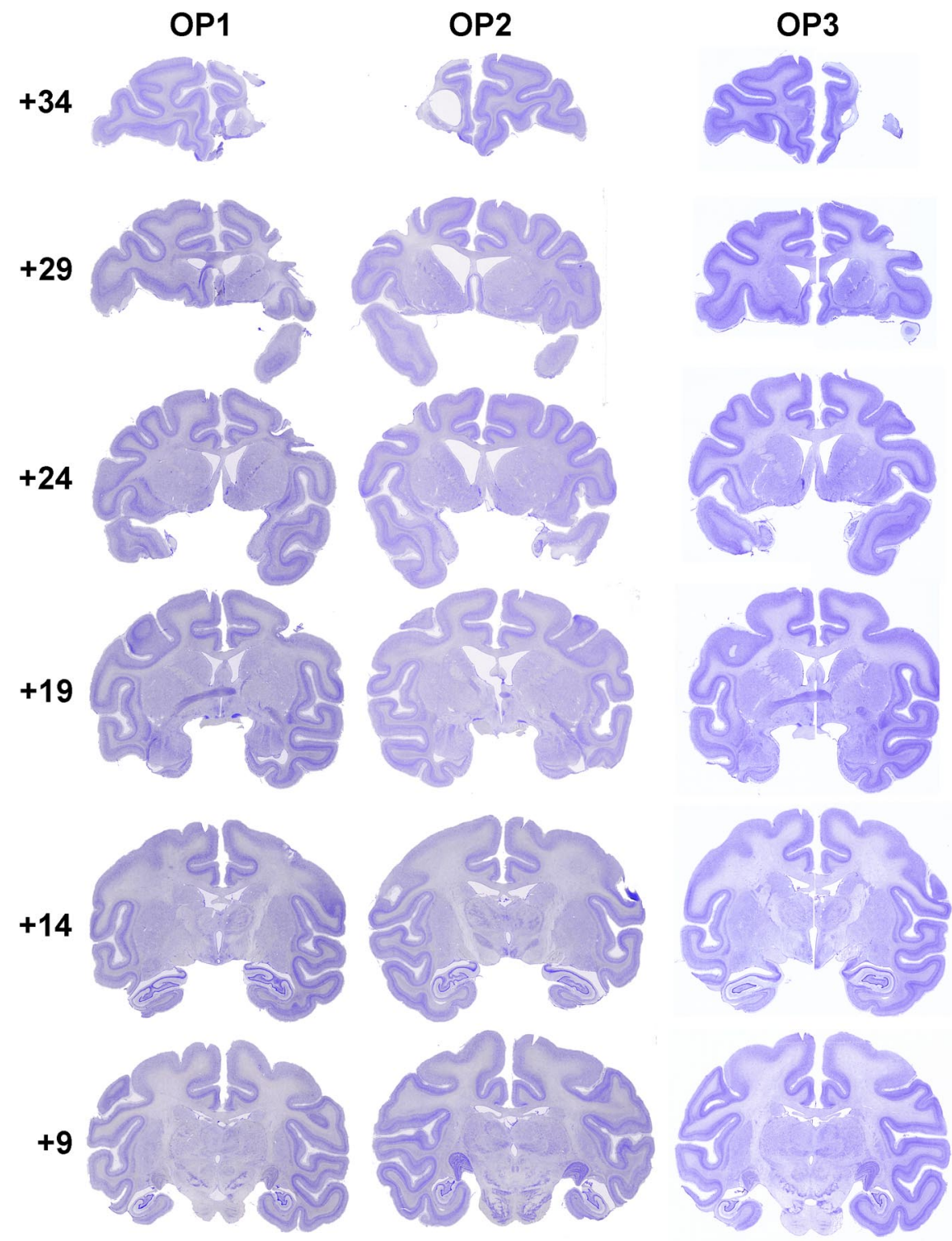

Figure 2. Histology. Coronal sections of monkeys from Group PFCXIT at different anterior-posterior levels (estimated from Paxinos et al., 2008). Monkey OP2 had left hemisphere prefrontal ablation and right hemisphere inferior temporal ablation. Monkeys OP1 and OP3 had right hemisphere prefrontal ablation and left hemisphere inferior temporal ablation.

from the cranium and retracted. A bone flap was raised and extended with a rongeur and then the dura mater was cut. The extent of the intended lesion is shown in Figure 1. The ablation extended from the fundus of the superior temporal sulcus to the fundus of the rhinal sulcus, and posteriorly included both banks of the anterior part of the occipitotemporal sulcus. The posterior limit of the ablation was a line drawn 


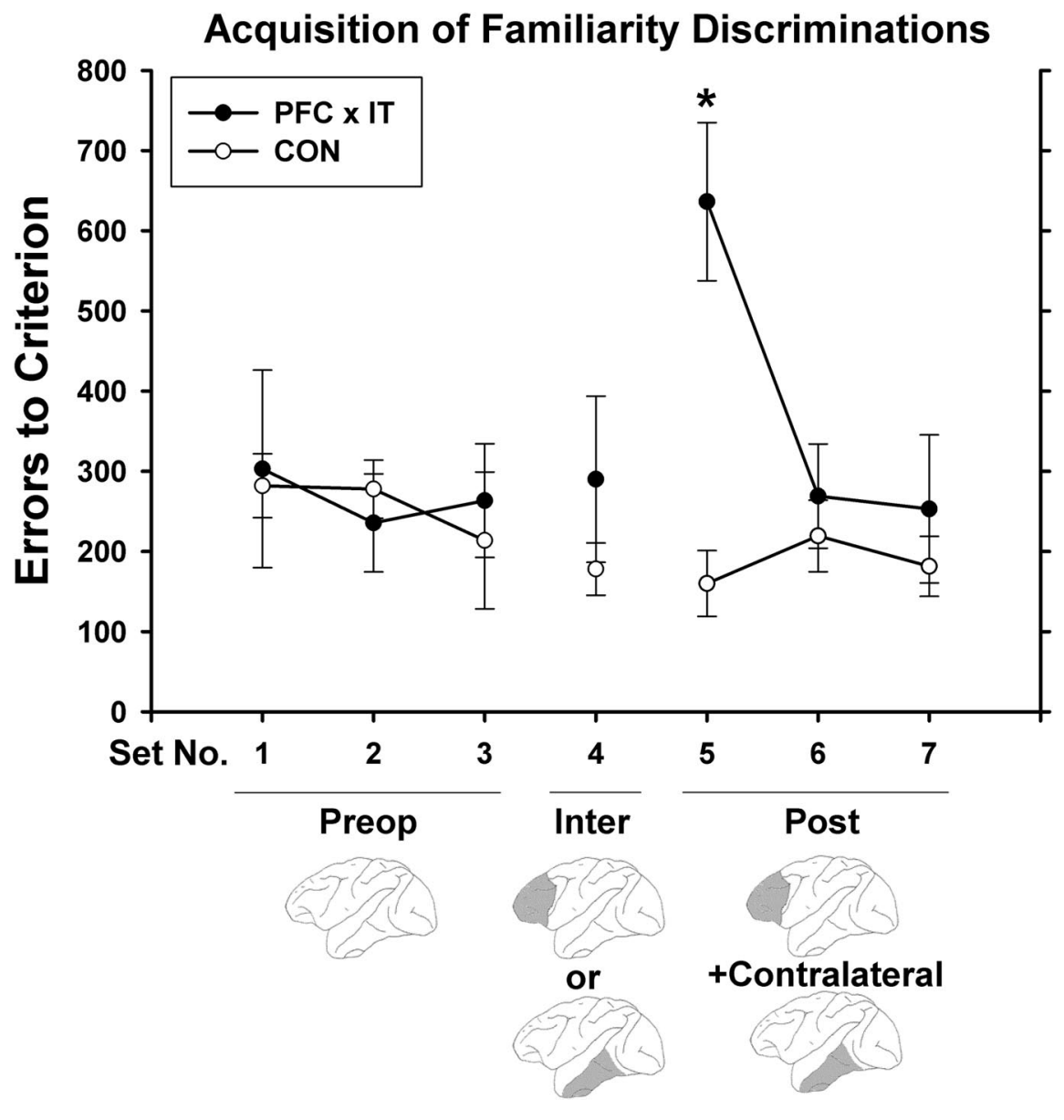

Figure 3. Experiment 1. Errors to criterion per set of 100 familiarity discriminations in the constant negative paradigm. Monkeys learned seven sets in total; three preoperative (Preop, sets 1-3), one interoperative (Inter, set 4), and three postoperative (Post, sets 5-7). Monkeys were unoperated during preoperative testing (Preop). Group PFCXIT had unilateral surgery to remove prefrontal cortex or inferotemporal cortex before interoperative testing (Inter). By the postoperative testing stage (Postop) Group PFCxIT had a second surgery to complete the PFC-IT disconnection. Monkeys were impaired only on set 5, the first postoperative set. Once the rule was reacquired, monkeys learned new familiarity discriminations at a rate comparable to controls.

perpendicular to the superior temporal sulcus, $5 \mathrm{~mm}$ anterior to the inferior occipital sulcus. The anterior limit of the ablation was the anterior tip of the superior temporal sulcus and a line drawn round the pole from that tip to the rhinal sulcus. Within these limits all the cortex was removed including both banks of the anterior and posterior middle temporal sulci.

\section{Histology}

At the completion of all behavioral testing, subjects were deeply anesthetized and transcardially perfused with physiological saline followed by $10 \%$ formalin. The brains were extracted and cut on a freezing microtome in $50 \mu \mathrm{m}$ sections in the coronal plane. The brains were extracted and cut on a freezing microtome in $50 \mu \mathrm{m}$ sections in the coronal plane. Every fifth section was stained with cresyl violet, mounted on a slide, and coverslipped. Figure 2 shows six coronal sections from each operated monkey (OP1-3) taken at $5 \mathrm{~mm}$ intervals. In all operated monkeys the prefrontal ablation included the entire orbitofrontal cortex, ventrolateral PFC, and dorsolateral PFC. The white matter surrounding the striatum and the striatum itself was intact. There was no striatal damage dorsal to the orbitofrontal lesion, or medially from the ablated principalis in any monkey. In monkey OP2 there was minor unintended damage to the anterior-most part of the descending limb of the arcuate sulcus. In monkey OP3 there was minor unintended damage to the dorsal bank of the cingulate sulcus at the anterior-most $3 \mathrm{~mm}$ of this sulcus.

In all three cases the inferotemporal ablation included the entire middle temporal gyrus, anterior middle temporal sulcus and perirhinal cor- tex, with the exception of OP2 in which the posterior $3 \mathrm{~mm}$ of medial perirhinal cortex was spared. There was slight unintended damage to lateral TF at its boundary to area TE, extending $2 \mathrm{~mm}$ medially to the occipitotemporal sulcus in each monkey. OP1 sustained $6 \mathrm{~mm}$ of damage in the AP plane to lateral TF, OP2 sustained $7 \mathrm{~mm}$ and OP3 sustained $5 \mathrm{~mm}$. In addition, the IT ablation in OP2 encroached upon area TEO by $2 \mathrm{~mm}$ posterior to the posterior limit of TE (TE, TF, and TEO of von Bonin and Bailey, 1947).

\section{Results}

Experiment 1

The acquisition and retention scores for Experiment 1 are displayed in Table 1. The number of errors to criterion was compared across the seven sets of problems learned using repeated-measures ANOVA with group as the betweensubjects factor. There was a main effect of Set $\left(F_{(1,6)}=14.49, p<0.001\right)$ and a group-by-set interaction $\left(F_{(1,6)}=27.81\right.$, $p<0.001)$ indicating that differences between sets were dependent on group. T tests between groups for each set indicated that set 5 was the only set in which the number of errors differed between groups (set 5: $\left.F_{(1,6)}=79.35, p<0.001\right)$. The error rates in all the remaining sets were not significantly different between groups. Preoperative learning rates were appropriately matched (set $1, F_{(1,6)}=0.11$; set 2 , $F_{(1,6)}=1.33$; set $\left.3, F_{(1,6)}=0.66 ; p>0.05\right)$. Unilateral surgery did not have any effect on learning rates ( set $4, F_{(1,6)}=4.79, p>$ 0.05 ) Learning rates for postoperative sets 6 and 7 were similar to those of controls $\left(\operatorname{set} 6, F_{(1,6)}=1.47 ; \operatorname{set} 7, F_{(1,6)}=2.06 ; p>\right.$ $0.05)$. Thus, following the severe impairment on set 5, the first new set acquired postoperatively, subsequent learning rates between the operated and control groups were equivalent. The impairment in learning set 5 is clearly shown in Figure 3 and the subsequent normalization of learning rates in the operated group can be seen in sets 6 and 7. After reacquisition of the task rule monkeys with prefrontal-temporal disconnection were able to learn new constant negative problems at a rate comparable to controls.

In addition to a retrograde amnesia for the task rule monkeys displayed a severe retrograde amnesia for preoperatively learned problems, in line with previous studies. Retention was assessed at 4 stages of the experiment; preoperatively (Preop), after unilateral surgery (Inter-op), after disconnection (Postop) and at the end of the experiment (Final). Both the main effect of Stage $\left(F_{(1,2)}=44.21, p<0.001\right)$ and Stage by Group interaction $\left(F_{(1,2)}=81.64, p<0.001\right)$ were highly significant. Pairwise comparisons revealed no differences between groups in Preop or Inter-op retention scores (Preop: $F_{(1,6)}=0.131$, Inter-op: $F_{(1,6)}=$ 0.023 ) indicating that unilateral surgery had no effect on retention scores. Comparisons revealed highly significant group differences in the Postop and Final retention scores (Postop: $F_{(1,6)}=57.47, p<$ 0.001 , Final: $\left.F_{(1,6)}=36.48, p=0.002\right)$. Overall therefore, there was a severe retrograde amnesia immediately postoperatively following 
PFC-IT disconnection. This amnesia persisted even after reacquisition of the task rule as evidenced by the persistence of the deficit in the Final retention stage.

\section{Experiment 2}

The data from Experiment 2 are shown in Figure 4. The acquisition of DNMS was impaired in monkeys with prefrontaltemporal disconnection with operated monkeys scoring $\sim 3$ times as many errors as controls to reach criterion $\left(F_{(1,6)}=\right.$ $8.64, p=0.032)$. This impairment appeared despite the fact that in Experiment 1 disconnected monkeys had already reacquired the task rule that novelty is always rewarded. Percentage correct scores of group PFCxIT were significantly worse than controls at each list length (LL) tested $\left(\mathrm{LL} 2, F_{(1,6)}=32.57, p=0.002\right.$; LL3, $F_{(1,6)}=28.89, p=0.003$; LL5, $F_{(1,6)}=$ $7.44, p=0.041)$. There was no LL $\times$ group interaction indicating that the size of the impairment did not depend on the number of objects that had to be remembered $\left(F_{(1,2)}=0.126\right)$. Across the LL performance tests, group PFCxIT were also impaired at each list position (LP), except LP5 (LP1, $F_{(1,6)}=12.06, p=0.018$; LP2, $F_{(1,6)}=21.57, p=0.006$; LP3, $F_{(1,6)}=$ $6.90, p=0.047$; LP4, $F_{(1,6)}=12.68, p=$ 0.016; LP5, $\left.F_{(1,6)}=3.76, p=0.110\right)$. An effect of LP approached significance $\left(F_{(1,4)}=2.67, \mathrm{df}=4, p=0.06\right)$ suggesting that the increased interference at distal LPs made the task harder. But there was no LP $\times$ group interaction $\left(F_{(1,4)}=0.588\right.$, $p=0.675)$ indicating that this effect did not modulate the size of the impairment.

\section{Discussion}

In this study, we tested monkeys with PFC-IT disconnection on two behavioral tasks to assess recognition memory, the constant negative task, and DNMS. The constant negative task was acquired preoperatively and required monkeys to discriminate familiar and novel objects, with unrewarded familiar objects presented once per day paired with rewarded novel objects that had never been presented before. These experiments produced two main findings. First, the process of discriminating familiar from novel objects, essential to normal performance of constant negative, was intact following reacquisition of the task rule. Second, the same monkeys were severely impaired in DNMS acquisition and were consistently and stably impaired in DNMS performance tests using longer lists of objects, even after the DNMS rule had been acquired. Therefore, operated monkeys were impaired in performing the DNMS task despite having demonstrated intact knowledge of the nonmatching rule and the ability to discriminate familiarity. This overall pattern of results supports our general hypothesis, laid out in the introduction, that one component of the deficits which result from lesions that include the prefrontal cortex is a deficit in the representation of temporally complex events. That familiarity recognition can proceed normally in monkeys with PFC-IT disconnection suggests that deficits in recognition memory tasks following PFC damage more likely stem from the temporally complex nature of the tasks.

The immediate postoperative retention deficit of preoperatively learned problems (sets 1-3) following PFC-IT disconnection was very severe. This deficit is likely partly due to retrograde amnesia for the nonmatching rule, as evidenced by impaired new learning on the first new postoperative set (set 5). However this deficit remained severe even after reacquisition of the rule (Table 1, Retention Sets, final). This suggests that the retrograde deficit following PFC-IT disconnection is not selective for task rules and must include information about specific visual object memories. This finding is supported by the fact that the retention of simple object-reward associative memories is severely impaired after this surgery (Browning and Gaffan, 2008b).

Monkeys may use a number of strategies when solving problems in the constant negative task. Our hypothesis is that learning in this task, like learning in DNMS, is driven predominantly by a strategy of responding to novel objects, identified by discriminating them from familiar objects (familiarity and novelty being memory-retrieved properties of objects) (Gaffan, 1985). However, another possible strategy is one based on "habit" learning 
(Mishkin and Petri, 1984). On this view, monkeys learn to avoid the constant negatives not because they are remembered as being familiar but because they are consistently associated with nonreward. In this case, correctly responding to the novel object does not represent an attraction to novelty but amounts to an exploration of a possible less bad option (the novel object has no reward history). We find it unlikely that monkeys would not use familiarity and novelty cues to guide responding. The rates of learning steadily displayed in the constant negative task greatly exceed those of traditional habit-based tasks, such as concurrent object discrimination learning. For example, using an identical testing apparatus and similar stimulus material (Gaffan et al., 2001, their Table 4) reported average rates of 4.37 errors per problem over 5 sets of 10 problem pairs of concurrent object discrimination learning. In constant negative control monkeys learned at a rate of 2.16 errors per problem over 7 sets of 100 problems per set, suggesting that familiarity discrimination mechanisms significantly contribute to learning in this task. Although we cannot definitively rule it out we find it highly unlikely that habit learning alone can account for the powerful learning exhibited in the constant negative task. In any case, this issue does not obviate our general hypothesis, namely that DNMS is impaired following PFC-IT disconnection by virtue of the temporal complexity of the task. Any associative strategy available in constant negative is similarly available in DNMS, so an explanation based around the temporal differences between the tasks must still be put forward.

The performance of familiarity discriminations was unimpaired by PFC-IT disconnection. This behavior is therefore likely directly supported by neurons in IT that modulate their signals in response to repeated presentations of objects (Brown and Xiang, 1998). This IT-dependent process is not sufficient for the normal performance of DNMS, as shown in Experiment 2. Our interpretation is that PFC-IT interactions support DNMS because performance can be greatly facilitated by representations, laid down in the sample phase, of what the appropriate choice should be at the upcoming choice phase, a temporally complex event. In contrast, in constant negative, there are no proximal cues that can be used to facilitate performance and so learning remains unimpaired after PFC-IT disconnection. The dissociation shown in this study between temporally complex and nontemporally complex recognition memory tasks complements previous dissociations, outlined in the introduction, between temporally complex and nontemporally complex object-reward association learning and reversal learning (Browning et al., 2007; Wilson and Gaffan, 2008).

In previous studies on the neural substrates of recognition memory, monkeys with perirhinal cortex lesions, mediodorsal thalamic lesions, or ventromedial prefrontal lesions display DNMS deficits that are modulated by the memory demands of the task, thus reflecting a true impairment of recognition memory (Aggleton and Mishkin, 1983; Bachevalier and Mishkin, 1986; Meunier et al., 1993). In contrast monkeys with lesions of the prefrontal inferior convexity do not display this pattern despite remaining impaired in reacquisition and performance of DNMS (Kowalska et al., 1991). Those impairments were therefore interpreted in terms of disorganized behavior rather than in terms of recognition memory. The pattern displayed by monkeys with prefrontal-temporal disconnection in this study more closely resembles that of monkeys with lesions of the inferior convexity of the prefrontal cortex. This pattern can be seen in Figure 4; increasing list length did not increase the impairment and there was no statistical effect of list position. These observa- tions, combined with Experiment 1, which showed that PFC-IT disconnection does not disrupt familiarity discrimination, underscore the fact that disconnected monkeys have difficulty making choices dependent on information presented in close temporal proximity within a particular trial. The impairment in DNMS with longer lists may be characterized as a deficit in updating the representation of a temporally complex event.

Theories of prefrontal function based on the temporal integration or assimilation of information as opposed to simply short-term memory are not new. Fuster (1997) described prefrontal function in terms of facilitating linkages within the perception-action cycle of behavior. Pribram and Tubbs (1967), in a series of elegant experiments in which prefrontal impairments on spatial alternation were ameliorated simply by increasing the length of the delay between each left-right sequence, noted that "[the] parsing of the stream of stimuli to which the organism is subjected, is a more important variable in the mechanism of short term memory than is the maintenance of the neural trace per se." We believe that DNMS with lists of objects particularly challenges monkey's ability to parse stimuli and thereby distinguish particular samples from particular choices.

Recognition memory depends on a widespread network of diencephalic, temporal, and frontal cortical areas. In humans, damage to the medial temporal lobe, diencephalic memory structures, and to some extent prefrontal cortical structures all cause deficits in judgments of prior occurrence. Lesion studies in monkeys have primarily relied on the delayed nonmatching to sample task for assessing recognition memory. The advantage of the DNMS paradigm is the ease with which assessments of memory load and duration can be performed. DNMS, however, relies on multiple cognitive processes, which in monkeys are difficult to dissociate. The DNMS task potentially relies not only on recognition memory but on object identification, episodic memory retrieval, short-term working memory, prospective memory, and rule implementation all working in concert to optimize task performance. The constant negative task represents another tool in assessing recognition memory in monkeys and may potentially be more useful as a specific probe of temporal lobe function because it can be performed without help from prefrontally based short term cognitive processes. It may be very useful to assess neuropsychological function in brain disease and aging, by dissociating familiarity discrimination/novelty detection processes from other aspects of recognition memory.

\section{References}

Aggleton JP, Mishkin M (1983) Visual recognition impairment following medial thalamic lesions in monkeys. Neuropsychologia 21:189-197. CrossRef Medline

Bachevalier J, Mishkin M (1986) Visual recognition impairment follows ventromedial but not dorsolateral prefrontal lesions in monkeys. Behav Brain Res 20:249-261. CrossRef Medline

Brown MW, Xiang JZ (1998) Recognition memory: neuronal substrates of the judgement of prior occurrence. Prog Neurobiol 55:149-189. CrossRef Medline

Browning PG, Gaffan D (2008a) Prefrontal cortex function in the representation of temporally complex events. J Neurosci 28:3934-3940. CrossRef Medline

Browning PG, Gaffan D (2008b) Global retrograde amnesia but selective anterograde amnesia after frontal-temporal disconnection in monkeys. Neuropsychologia 46:2494-2502. CrossRef Medline

Browning PG, Easton A, Buckley MJ, Gaffan D (2005) The role of prefrontal cortex in object-in-place learning in monkeys. Eur J Neurosci 22:32813291. CrossRef Medline

Browning PG, Easton A, Gaffan D (2007) Frontal-temporal disconnection abolishes object discrimination learning set in macaque monkeys. Cereb Cortex 17:859-864. CrossRef Medline 
Browning PG, Gaffan D, Croxson PL, Baxter MG (2010) Severe scene learning impairment, but intact recognition memory, after cholinergic depletion of inferotemporal cortex followed by fornix transection. Cereb Cortex 20:282-293. CrossRef Medline

Bussey TJ, Wise SP, Murray EA (2002) Interaction of ventral and orbital prefrontal cortex with inferotemporal cortex in conditional visuomotor learning. Behav Neurosci 116:703-715. CrossRef Medline

Fuster JM (1997) The prefrontal cortex: anatomy, physiology and neuropsychology of the frontal lobe, Ed 3. Philadelphia: Lippincott-Raven.

Fuster JM, Bauer RH, Jervey JP (1985) Functional interactions between inferotemporal and prefrontal cortex in a cognitive task. Brain Res 330:299307. CrossRef Medline

Gaffan D (1985) Hippocampus: memory, habit and voluntary movement. Philos Trans R Soc Lond B Biol Sci 308:87-99. CrossRef Medline

Gaffan D, Parker A, Easton A (2001) Dense amnesia in the monkey after transection of fornix, amygdala and anterior temporal stem. Neuropsychologia 39:51-70. CrossRef Medline

Gaffan D, Easton A, Parker A (2002) Interaction of inferior temporal cortex with frontal cortex and basal forebrain: double dissociation in strategy implementation and associative learning. J Neurosci 22:7288-7296. Medline

Kowalska DM, Bachevalier J, Mishkin M (1991) The role of the inferior prefrontal convexity in performance of delayed nonmatching-to-sample. Neuropsychologia 29:583-600. CrossRef Medline

Meunier M, Bachevalier J, Mishkin M, Murray EA (1993) Effects on visual recognition of combined and separate ablations of the entorhinal and perirhinal cortex in rhesus monkeys. J Neurosci 13:5418-5432. Medline
Mishkin M, Petri, HL (1984) Memories and habits: some implications for the analysis of learning and retention. In: Neuropsychology of memory (Squire NBaLR, ed), pp 287-296. New York: Guilford.

Murray EA, Gaffan D (2006) Prospective memory in the formation of learning sets by rhesus monkeys (macaca mulatta). J Exp Psychol Anim Behav Process. 32:87-90. CrossRef Medline

Parker A, Gaffan D (1998a) Interaction of frontal and perirhinal cortices in visual object recognition memory in monkeys. Eur J Neurosci 10:30443057. CrossRef Medline

Parker A, Gaffan D (1998b) Memory after frontal/temporal disconnection in monkeys: conditional and non-conditional tasks, unilateral and bilateral frontal lesions. Neuropsychologia 36:259-271. CrossRef Medline

Paxinos G, Huang XF, Toga AW (2008) The rhesus monkey brain in stereotaxic coordinates. San Diego: Academic.

Pribram KH, Tubbs WE (1967) Short-term memory, parsing, and the primate frontal cortex. Science 156:1765-1767. CrossRef Medline

Tanaka K (1996) Inferotemporal cortex and object vision. Annu Rev Neurosci 19:109-139. CrossRef Medline

von Bonin G, Bailey P (1947) The neocortex of Macaca mulatta. Urbana, IL: University of Illinois.

Wilson CR, Gaffan D (2008) Prefrontal-inferotemporal interaction is not always necessary for reversal learning. J Neurosci 28:5529-5538. CrossRef Medline

Wilson CR, Gaffan D, Browning PG, Baxter MG (2010) Functional localization within the prefrontal cortex: missing the forest for the trees? Trends Neurosci 33:533-540. CrossRef Medline 